\title{
Disulfide bond formation is not crucial for the heat-induced interaction between $\beta$-lactoglobulin and milk fat globule membrane proteins
}

\author{
Steffen F. Hansen, ${ }^{1}$ Søren D. Nielsen, ${ }^{1}$ Jan T. Rasmusen, ${ }^{2}$ Lotte B. Larsen, ${ }^{1}$ and Lars Wiking ${ }^{1 *}$ \\ ${ }^{1}$ Department of Food Science, Aarhus University, Agro Food Park 48, 8200 Aarhus N, Denmark \\ ${ }^{2}$ Department of Molecular Biology and Genetics, Aarhus University, Gustav Wieds Vej 10C, 8000 Aarhus C, Denmark
}

\begin{abstract}
During heat treatment of milk, $\beta$-lactoglobulin $(\beta$ LG) associates with the milk fat globule membrane (MFGM). The objective of this study was to examine different binding types that could be involved in this process. First, we tested the thiol-disulfide bond interchange between $\beta$-LG and MFGM by heating raw milk $\left(87^{\circ} \mathrm{C}, 8 \mathrm{~min}\right)$ in the presence of different reagents capable of preventing this interaction, and then evaluated the presence of $\beta$-LG in resulting MFGM preparations by sodium dodecyl sulfate-PAGE. Contrary to commonly accepted theory, $\beta-\mathrm{LG}$ still associated with MFGM when milk was heated in the presence of 10 $\mathrm{m} M N$-ethylmaleimide, dithiobis-nitrobenzoic acid, or dithioerythritol. This finding indicated that noncovalent binding could be involved in the interaction, and therefore these were studied next. Preventing noncovalent interactions by heating milk in the presence of $8 M$ urea (to inhibit formation of hydrogen bonds) or $2 M$ $\mathrm{NaCl}$ (to inhibit electrostatic and hydrophobic interactions) reduced the association of $\beta-\mathrm{LG}$ and MFGM. Inhibiting both hydrogen and disulfide bond formation by addition of $8 M$ urea and $10 \mathrm{~m} M$ dithioerythritol or inhibiting hydrophobic interactions with $0.2 \%$ sodium dodecyl sulfate completely prevented the association. In contrast to the simple thiol-disulfide interaction model, the results suggest a more complex understanding of the interactions between $\beta-\mathrm{LG}$ and MFGM during heating of milk. This indicates that disulfide formation between $\beta-\mathrm{LG}$ and proteins in the MFGM is not required for the association, but that hydrophobic interactions and hydrogen bonding may be crucial. This novel insight into $\beta$-LG and MFGM association is in contrast to the current literature and requires further study.
\end{abstract}

Key words: milk fat globule membrane, interaction, $\beta$-lactoglobulin, processing, thermal treatment

Received December 16, 2019.

Accepted March 7, 2020.

*Corresponding author: lars.wiking@food.au.dk

\section{INTRODUCTION}

The milk fat globule membrane (MFGM) is an important component of bovine milk, as it serves to emulsify milk fat globules and protect them from lipolysis and oxidation. In recent years, the large content of polar lipids, most notably sphingomyelin, and bioactive proteins in MFGM has made it a value-added ingredient with both functional and nutritional benefits, for instance when used in infant formulas (Claumarchirant et al., 2016). The composition of MFGM is known to undergo a range of changes in response to various milk processing conditions. For instance, the release of MFGM fragments in response to mechanical stress during pumping and homogenization promotes the adsorption of casein micelles to fat globule surface and, hence, the remaining MFGM fragment; meanwhile, the presence of $\beta-\mathrm{LG}$ in MFGM material is caused by several heat treatment steps (Lee and Sherbon, 2002).

Depending on the intended use of the heat-treated milk, the association of $\beta$-LG to MFGM can be desired or undesired. It contributes to the texture and structure of cheese and yogurt (Fox et al., 2015), but it may impair the emulsifying properties of MFGM (Corredig and Dalgleish, 1998). It occurs in a dose-dependent manner in response to combinations of heating temperature and time, either at $87^{\circ} \mathrm{C}$ for $2.5 \mathrm{~min}$ (Kim and Jiménez-Flores, 1995) or $>60^{\circ} \mathrm{C}$ for $10 \mathrm{~min}$ (Ye et al., 2004 ). Because $\beta$-LG is only visible in SDS-PAGE gels of MFGM preparations upon reduction of all disulfide bonds, current consensus holds that the association of $\beta$-LG to milk fat globules is based on a thiol-disulfide interchange reaction between $\beta$-LG and MFGM proteins (Ye et al., 2004). However, the exact mechanism behind it is unknown. Alternative explanations include hydrophobic interactions between denatured $\beta-L G$ and MFGM lipids (Houlihan et al., 1992) and "more complex interactions" (Kim and Jiménez-Flores, 1995).

$\beta$-Lactoglobulin contains 2 internal disulfide/cystine groups and possesses a reactive, free thiol group at $\mathrm{Cys}^{121}$, which is normally buried in the interior of the molecule, but becomes exposed to the serum phase upon 
denaturation and unfolding of the $\beta$-LG molecule (Fox et al., 2015). Indeed, early experiments revealed that $\mathrm{Cys}^{121}$ is primarily responsible for formation of $\beta-\mathrm{LG}$ self-aggregates or complexes with other milk components through thiol-disulfide interchange, because $\beta$-LG treated with the thiol-blocking agent $N$-ethylmaleimide (NEM) did not form aggregates (Elfagm and Wheelock, 1978; Hong et al., 1984). Supporting this view, kinetics of $\beta$-LG self-aggregation correspond to that of thiol-disulfide interchange (Hoffmann et al., 1997). Other studies found that if $\mathrm{Cys}^{121}$ was made inert by NEM blocking, noncovalent interactions may also play a role in the aggregation of $\beta$-LG (Hoffmann and Van Mil, 1997; Kitabatake et al., 2001). Furthermore, internal disulfide groups of $\beta$-LG may also partake in aggregation if converted to thiol groups by gentle reduction before heating (Wada and Kitabatake, 2001).

Extrapolating this knowledge from homogeneous $\beta-L G$, whey protein concentrate solutions, or more complex systems such as whole milk, leads to the natural conclusion that $\mathrm{Cys}^{121}$ must also be the primary driving force behind $\beta$-LG and MFGM aggregation in response to heat treatments. Yet, to our knowledge, this has not been proven, and no studies have evaluated whether other covalent or noncovalent binding types, apart from disulfide bond formation, might be involved.

The aim of this study was to elucidate the heat-induced interaction between $\beta-L G$ and MFGM proteins. By heating whole milk in the presence of reagents that inhibit the formation of various binding types, we attempted to study the role of these different binding types by evaluating the prevalence of $\beta$-LG in MFGM material from the heated milk. Based on the consensus that thiol-disulfide bond interchange is the primary type of association, we hypothesized that heating in the presence of thiol-blocking agents-preventing the reactive $\mathrm{Cys}^{12}$ in $\beta$-LG from forming disulfide bonds to MFGM proteins - would prevent the formation of the $\beta$-LG-MFGM adducts. Additionally, a range of chaotropic reagents were tested to evaluate the role of noncovalent binding types in the association between $\beta$-LG and MFGM.

\section{MATERIALS AND METHODS}

\section{Chemicals}

Raw bovine milk was collected at the Danish Cattle Research Centre (Aarhus University, Tjele, Denmark). Liquid chromatography (LC)-MS-grade acetonitrile (ACN), DL-lanthionine (LAN) and D-lysine-4,4,5,5-d4 (internal standard) were purchased from Sigma-Aldrich Chemicals (St. Louis, MO), while LL- and LD-lysinoalanine $(\mathbf{L A L})$ in a diastereomeric mixture was procured from Bachem (Bubendorf, Switzerland). DL-Cystine$3,3,3^{\prime}, 3^{\prime}$-d4 (internal standard) was obtained from Cambridge Isotope Laboratories (Cambridge, MA). The LC-MS grade water was used in the triple Q measurements, and MilliQ water [produced in-house on a Milli-QR Reference Water Purification System (Merck Millipore, Darmstadt, Germany)] was used for all other solutions. $\quad N$-Ethylmaleimide, dithiobis-nitrobenzoic acid (DTNB), dithioerythritol (DTE), urea, SDS, sodium chloride, and all other chemicals were procured from Sigma-Aldrich.

\section{Preparation of MFGM Material}

Different reagents, each capable of preventing different types of interactions (Table 1), were dissolved in raw milk by stirring for $2 \mathrm{~h}$ at room temperature. Concentrations were based on previous studies by Kitabatake et al. (2001) and Martin et al. (2014). To induce denaturation and complexation between MFGM proteins and serum proteins, milk in $50-\mathrm{mL}$ tubes were transferred to an $87^{\circ} \mathrm{C}$ water bath for $8 \mathrm{~min}$, and subsequently cooled in ice. Unheated milk and heated milk without reagents were included as controls. Isolation of MFGM material followed the method of Gregersen et al. (2019). Briefly, the cream phase was separated from skim milk by centrifugation at $4^{\circ} \mathrm{C}$ for 30 min at 3,500 $\times g$ (Multifuge 3 S-R, Heraeus, Hanau, Germany), followed by washing in $0.05 \mathrm{M}$ phosphate buffer, $\mathrm{pH}$ 7 , and reseparation using the same centrifugation parameters. The washing step was repeated twice. The washed cream phase was transferred to Eppendorf tubes and pre-heated to $40^{\circ} \mathrm{C}$, and MFGM was pelleted by centrifugation at $19,000 \times g$ for $30 \mathrm{~min}$ at $40^{\circ} \mathrm{C}$. Finally, the pellet was resuspended in $100 \mu \mathrm{L}$ of water and subjected to protein content determination by a bicinchoninic acid assay (Thermo Scientific, Roskilde, Denmark). The overall isolation process is outlined in Figure 1.

\section{MFGM Protein Composition}

Resuspended MFGM material was subjected to SDSPAGE under both nonreducing and reducing conditions as described by Hansen et al. (2018). Briefly, samples were diluted to $1 \mu \mathrm{g} / \mu \mathrm{L}$ of protein in Laemmli sample buffer without or with $10 \mathrm{~m} M \mathrm{DTE}$ as reducing agent before heating to $95^{\circ} \mathrm{C}$ for $10 \mathrm{~min}$. Approximately 10 $\mu \mathrm{g}$ of protein was loaded into each well, and protein bands were visualized by the fluorescent response to UV irradiation. Each protein band was identified based on published gels (Gassi et al., 2016; Hansen et al., 2018), while bands of particular interest were scrutinized as described below. Densitometric measurements 
of each band intensity allowed the determination of relative content of $\beta$-LG to major MFGM proteins (i.e., xanthine oxidase, butyrophilin, and periodic acid Schiff base 6 and 7 , PAS 6/7).

\section{Identification of Individual Bands}

Bands expected to contain $\beta$-LG were digested in-gel and subjected to peptide mass fingerprinting against the SwissProt database by matrix-assisted laser desorption ionization-time of flight-mass spectrometry (MALDI-TOF-MS) using an Ultraflex tandem mass spectrometer (Bruker Daltonics, Bremen, Germany) as described by Larsen et al. (2010).

\section{Quantification of Isopeptide Bonds}

The MFGM preparations from whole milk unheated, heated, or heated in presence of NEM were analyzed for their content of LAL and LAN by LC-MS triple quadrupole operated in multiple reaction monitoring mode as follows. Samples were acid-hydrolyzed using 10 $N \mathrm{HCl}$ for $24 \mathrm{~h}$ at $110^{\circ} \mathrm{C}$. Afterward, they were diluted $10 \times$ in LC-MS grade water and centrifuged at 10,000 $\times g$ for $15 \mathrm{~min}$. The supernatant was then mixed with $1 \mathrm{M} \mathrm{NaOH}$. The 2 internal standards were spiked into the mixture before the samples were loaded onto the LC-MS. Samples were then separated on an Intrada WAA35 Amino Acid column (Imtakt, Kyoto, Japan) fitted in a 1260 Infinity LC system coupled to a 6460 QQQ mass spectrometer (Agilent Technologies, Waldbronn, Germany) with mobile phases containing (A) $100 \mathrm{~m} M$ of ammonium formate and (B) $90 \% \mathrm{ACN}$ in $0.1 \%$ solution of formic acid. The following gradients were used at $600 \mu \mathrm{L} / \mathrm{min}$ flow rate: $25-100 \% \mathrm{~A}(3 \mathrm{~min})$, followed by $100 \%$ A (5 min), and finally $25 \%$ A (2 $\min$ ) to re-equilibrate the instrument. Q1/Q3 masses of LAL, LAN, and internal standards were used to collect data, which were quantified using ratios of analyte to internal standard in MassHunter Quantitate Analysis software (Agilent Technologies). Using serial standard dilutions, the limit of detection was established to be $1.8 \mathrm{ppm}$ and $0.09 \mathrm{ppm}$ for LAL and LAN, respectively.

\section{Statistics}

All experiments were conducted in duplicate or triplicate. When relevant, relative $\beta$-LG contents were compared by one-way ANOVA (Duncan's multiple range test) at a significance level of $P<0.05$ in R (v. 3.1.1; https://www.R-project.org/).

\section{RESULTS AND DISCUSSION}

\section{Effect of Thiol-Blocking and Reducing Agents}

Whole milk was heated $\left(87^{\circ} \mathrm{C}, 8 \mathrm{~min}\right)$ in the presence of thiol-blocking agents (NEM, DTNB) or reducing agent (DTE) to evaluate the ability of these agents to prevent the association of $\beta$-LG with MFGM. Figure 2 shows no $\beta-L G$ in unheated MFGM preparations (lane 6 ), but $\beta$-LG present in heated preparations under reducing conditions (lane 7). No $\beta$-LG was detected in heated, nonreduced samples (lane 2), which supports the consensus that disulfide bonds are responsible for the complexation between $\beta$-LG and MFGM (Ye et al., 2004).

Contrary to our hypothesis, heating in presence of NEM still resulted in $\beta$-LG association to MFGM (lane 8), despite the ability of NEM to block the reactive thiol group of $\mathrm{Cys}^{121}$. Moreover, $\beta$-LG was detectable under nonreducing conditions (lane 3 ). To avoid any misinterpretation, all $\beta$-LG bands were positively identified by in-gel tryptic digestion, followed by peptide mass matching using MALDI-TOF-MS. The same pattern was observed for concentrations of near-unimolar

Table 1. Overview of reagents added to milk before heating and the different types of protein-protein interactions they allow $(+)$ or prevent $(-)^{1}$

Type of interaction possible

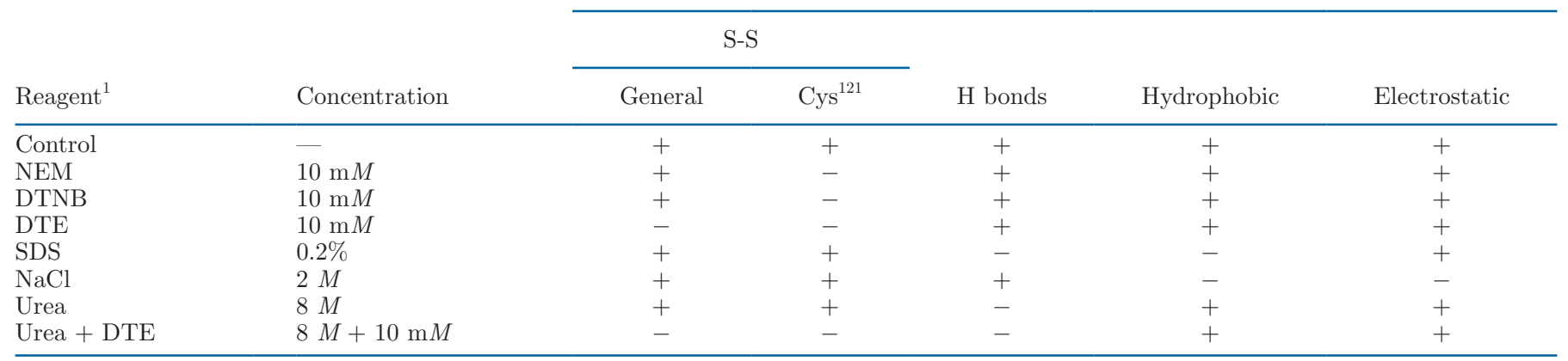

${ }^{1} \mathrm{NEM}=N$-ethylmaleimide; DTNB $=$ dithiobis-nitrobenzoic acid; DTE $=$ dithioerythritol. 


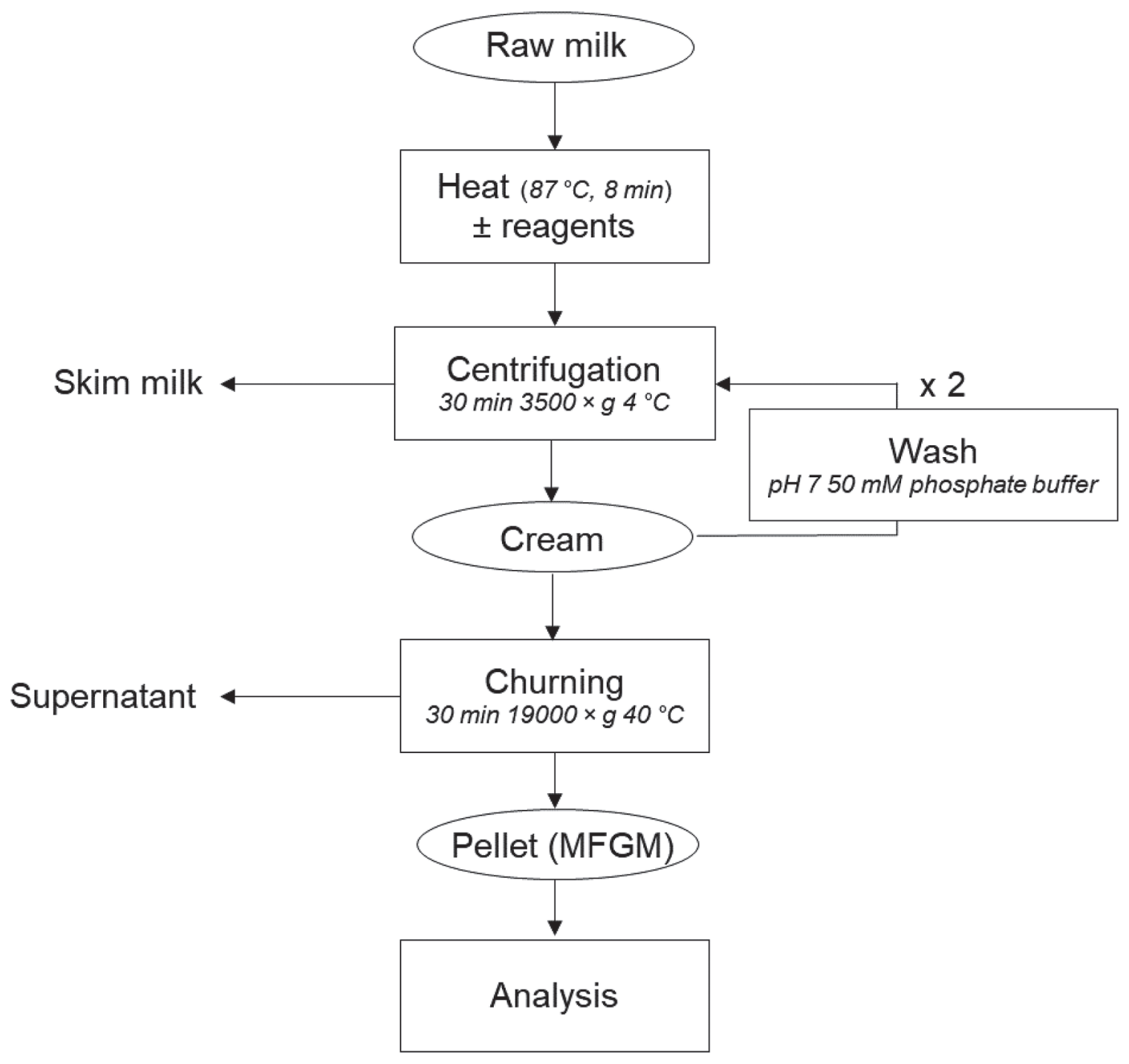

Figure 1. The procedure for obtaining milk fat globule membrane (MFGM) material. The reagents are listed in Table 1.

amounts of NEM to $\beta$-LG (0.4 mM; not shown). Adding NEM after heating (not shown) resulted in the same pattern as milk heated without NEM.

To confirm the observations in another system, an alternative thiol-blocking molecule, DTNB, was applied, and showed the same result: visible $\beta$-LG appeared in both nonreduced and reduced samples (lane 4 and 9 , respectively). This implies that $\beta$-LG still adsorb to MFGM in response to heating, even when lacking the reactive thiol group on $\mathrm{Cys}^{121}$.

To see if there could be a contribution from internal disulfide groups in $\beta$-LG, DTE was added to whole milk before heating at a concentration of $10 \mathrm{mM}$. This concentration is sufficient to rule out any possible thioldisulfide interchange reaction by complete reduction (Wada and Kitabatake, 2001). Nonetheless, the level of association of $\beta$-LG to MFGM was unchanged and visible under both reducing and nonreducing conditions (lane 5 and 10), suggesting that the internal disulfide groups of $\beta$-LG did not contribute to $\beta$-LG-MFGM complexation at the conditions tested. Adding DTE after heating did not prevent association of $\beta-\mathrm{LG}$ with MFGM, although some $\beta$-LG appeared in nonreduced samples and, hence, must have been adsorbed to MFGM by means other than disulfide bonds.

On a side note, the intensity of $\beta$-LG in lanes $4,5,9$, and 10 was more pronounced than in lanes 3,7 , and 8 . The exact reason for this is not known, but it is possible that solvent compositional changes after DTNB and DTE addition make the adsorption of $\beta$-LG to MFGM more favorable. The mass difference observed between $\beta$-LG in lane 6 and both lanes 4 and 5, was probably due to the fact that $\beta$-LG heated in presence of NEM and DTNB would still have intact internal disulfide bridges and, thus, maintain $\beta-\mathrm{LG}$ in a more compact and electrophoretically mobile conformation 
(lane 4 and 5) than the fully unfolded DTE sample, which would be less mobile (lane 6). Alternatively, the displaced bands could be due to complexes of $\beta-\mathrm{LG}$ and NEM/DTNB, which were identified by Wijayanti et al. (2015) using electrospray ionization/time-of-flight MS.

The ratio between $\beta$-LG intensity in unreduced vs. reduced sample buffers was close to zero for the MFGM preparation from milk heated in absence of reagents. A classical interpretation of this was that $\beta$-LG must be trapped in supermolecular complexes in the top of the gel by disulfide bonds. In contrast, when milk was heated in presence of either NEM, DTNB or DTE, the ratio was close to 1 . This further solidifies that disulfide bond formation play an insignificant role in these

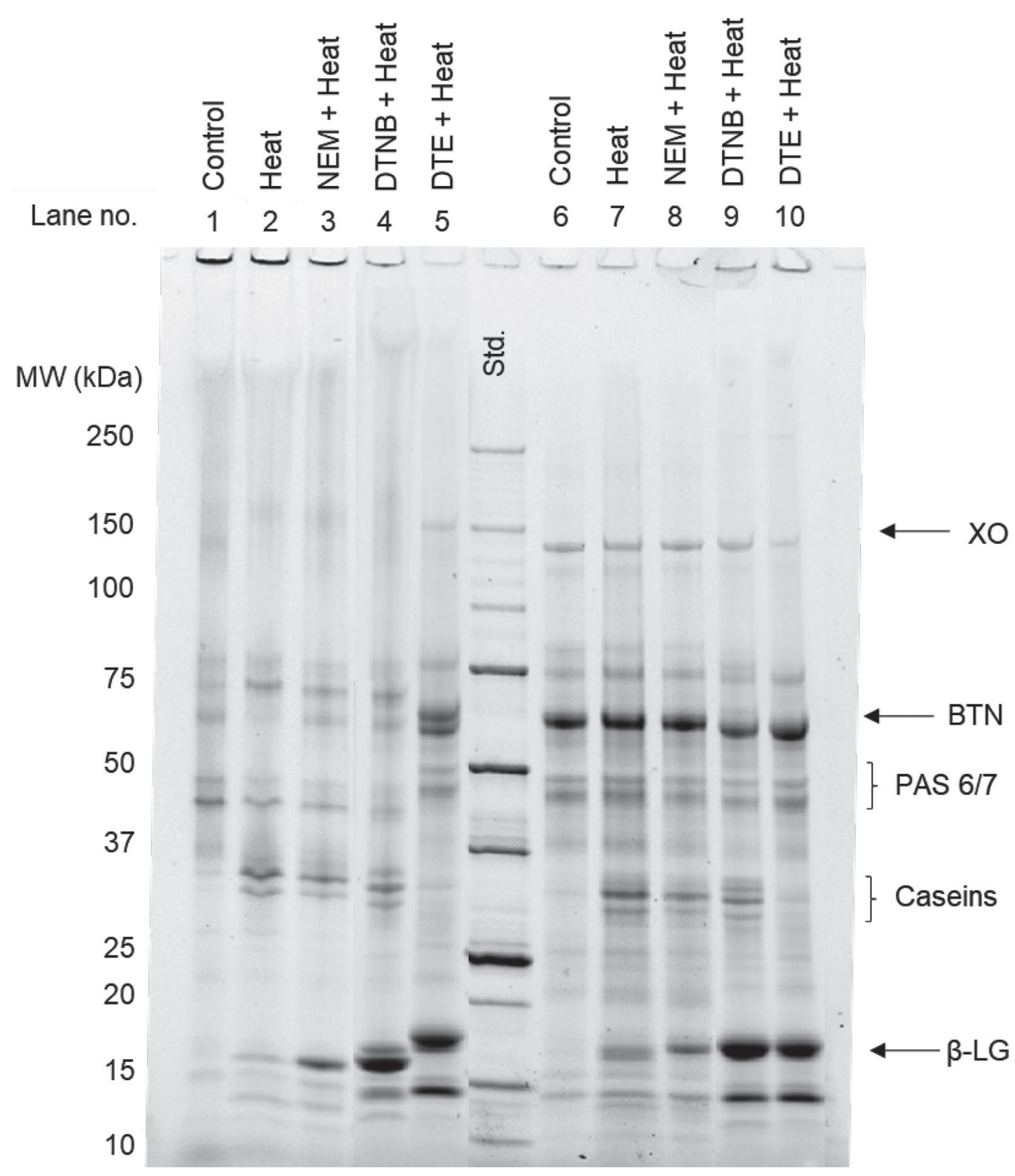

Figure 2. Effect of thiol-blocking reagents (10 $\mathrm{m} M$ NEM or DTNB) and reducing agent (10 $\mathrm{m} M$ DTE) on heat-induced $\beta$-LG content of milk fat globule membrane (MFGM) material. A representative SDS-PAGE gel is shown with nonreduced (lanes 1-5) and reduced (lanes 6-10) MFGM material. The MFGM proteins were identified based on their electrophoretic mobility compared with earlier studies, and the identity of $\beta$-LG was confirmed by in-gel tryptic digestion followed by MALDI-TOF MS peptide mass matching. MW = molecular weight; Std. = molecular weight standard; $\mathrm{XO}=$ xanthine oxidase; BTN = butyrophilin; PAS $6 / 7=$ periodic acid Schiff base 6 and $7 ; \mathrm{NEM}=N$-ethylmaleimide; DTNB $=$ dithiobis-nitrobenzoic acid; DTE $=$ dithioerythritol. 
samples. In a simple $\beta$-LG suspension, a study showed that NEM addition before heating resulted in $\beta-L G$ aggregates, and this intermolecular interaction, in lieu of disulfide bond formation, was ascribed to unspecified noncovalent interactions. (Hoffmann and Van Mil, 1997). In contrast, a pure solution of $\beta$-LG molecules adducted to NEM did not form aggregates upon heating (Kitabatake et al., 2001).

The results indicate that complexation between $\beta$-LG and MFGM can occur by other means than thioldisulfide group interchange when this option is blocked by NEM, DTNB, or DTE. Could the alternative interaction be another type of covalent bond induced by heating, for example, isopeptide or Maillard reaction products? The total amount of isopeptide bonds (i.e., primary products of isopeptide bonds, LAL, and LAN; Wada and Lönnerdal, 2015) in the MFGM preparations was quantified and found to be below detection limit for both LAL and LAN, regardless of heating (data not shown). Thus, the formation of isopeptide bonds between $\beta$-LG and MFGM proteins in response to heating was ruled out. Maillard reactions could theoretically occur between the $\mathrm{N}$ terminus of $\beta-\mathrm{LG}$ and sugar moieties on the heavily glycosylated MFGM proteins, but we did not assess furosine, a Maillard marker molecule, in this study. After all, the detection of $\beta-L G$ in nonreduced SDS-PAGE would not be possible, if $\beta$-LG had been trapped in covalently bound complexes in the top of the gels. The results imply that noncovalent interactions might fully compensate for the lack of the reactive thiol group on $\mathrm{Cys}^{121}$ in the heat-induced interaction between $\beta-\mathrm{LG}$ and MFGM.

\section{Effect of Chaotropic Agents and Salt}

To specify which noncovalent interactions could be involved in the interaction, a range of chaotropic reagents - each interfering with one or more noncovalent protein-protein interactions - were added before heating of the whole milk. Heating milk in the presence of $\mathrm{NaCl}$ resulted in a significant $(P<0.05)$ reduction in relative content of MFGM-associated $\beta$-LG to $12 \pm 3.0 \%$ (Figure 3 , lane 9 ), compared with $35 \pm 7.3 \%$ in milk heated without any reagents (lane 8). This reduced level of $\beta-L G$ was assumed to be attached to MFGM through disulfide bonds, based on the absence of $\beta-\mathrm{LG}$ band in nonreduced sample (lane 3 ). It cannot be excluded that salt addition had a protective effect on $\beta$-LG toward heating due to the favoring of dimer configuration, as suggested by Kella and Kinsella (1988). Adding $\mathrm{NaCl}$ after heating had no effect on the level of $\beta$-LG (not shown), suggesting that once disulfide bonds to MFGM proteins were established, they could not be cancelled by manipulating the hydrophobic and electrostatic interactions. Thus, hydrophobic and electrostatic interactions, which are disfavored in the presence of $\mathrm{NaCl}$ (Martin et al., 2014), appear to play an important role in establishing the thiol-disulfide interchange reaction between MFGM proteins to $\beta$-LG.

Interestingly, a similar effect was observed by heating in the presence of urea (lane 10), where the relative $\beta$-LG content in MFGM preparations was also significantly reduced to $12 \pm 6.0 \%(P<0.05)$ when compared with control. Using the same argument as above, hydrogen bonds, which are disturbed by urea, must also be important for the association of $\beta$-LG to MFGM, and again, the formation of disulfide bonds appeared to be responsible for the remaining interaction. Indeed, if both hydrophobic interaction and disulfide bond formation were prevented by heating milk in the presence of urea and DTE, the result was an absence of $\beta$-LG in MFGM preparations (lane 11).

We found an equally pronounced effect with SDS, because it completely prevented any interaction between $\beta-L G$ and MFGM proteins (lane 12). Sodium dodecyl sulfate is known to disrupt both hydrogen bonds and hydrophobic interactions (Martin et al., 2014). In addition, the hydrophobic dodecyl tail of SDS associates with hydrophobic patches of denaturing proteins, thus introducing a net negative charge. Therefore, the thioldisulfide-based association of $\beta$-LG to MFGM proteins was probably also avoided by electrostatic repulsion between the proteins. Adding SDS after heating had no effect on the already induced association of $\beta$-LG (not shown).

The results imply that thiol-disulfide interchange reaction cannot be held solely responsible for $\beta-\mathrm{LG}$ association to MFGM, which was also suggested by (Kim and Jiménez-Flores, 1995). Rather, a complex combination of noncovalent interactions (hydrogen bonds, as well as hydrophobic and electrostatic interactions) appear to play equally important roles in establishing the right conditions for $\mathrm{Cys}^{121}$ of $\beta$-LG to react with cystines/cysteines in MFGM proteins. Alternative binding theories include hydrophobic interactions between $\beta$-LG and long side chains of membrane lipids upon heat-induced displacement of PAS 6/7 from MFGM (Houlihan et al., 1992), and electrostatic interaction between $\beta$-LG and charged sugar moieties of glycosylated MFGM proteins (Kim and Jiménez-Flores, 1995). Both theories are compatible with our findings, where disturbing electrostatic, hydrophobic, or hydrogen bonding reduced the association of $\beta$-LG to MFGM. The exact mechanism, however, remains a topic for future studies, where a model system of washed fat globules, or even individual MFGM proteins, and pure $\beta$-LG 
solutions could be subjected to individual or combined heating in presence of the reagents used in this study. For instance, heating of MFGM is known to cause a release of PAS 6/7 (Ye et al., 2004), and it would be relevant to investigate, if subsequent heating in presence of $\beta$-LG would result in a lesser extent of $\beta$-LG association and thereby point toward PAS $6 / 7$ as an important protein binding site for $\beta$-LG. The role of the casein micelle as a potential interaction mediator could also be considered, since $\beta$-LG can bind to $\kappa$-casein in response to heating (Oldfield et al., 2000), which can also bind to MFGM and thereby form a bridge between $\beta-L G$ and MFGM (Ye et al., 2004).

\section{CONCLUSIONS}

The potential interactions between $\beta$-LG and MFGM during heat treatment of milk was studied by stepwise ruling out various binding types that could be involved. Heating milk under normal conditions (control) lead to absorption of $\beta$-LG to MFGM. However, blocking the option of thiol-disulfide interactions between $\mathrm{Cys}^{121}$ on $\beta$-LG and disulfide bridges within MFGM proteins by NEM, DTNB, or DTE still lead to $\beta$-LG-MFGM adducts, which pointed toward the participation of noncovalent interactions. Indeed, disturbing hydrogen bonds (urea) or hydrophobic and electrostatic interactions

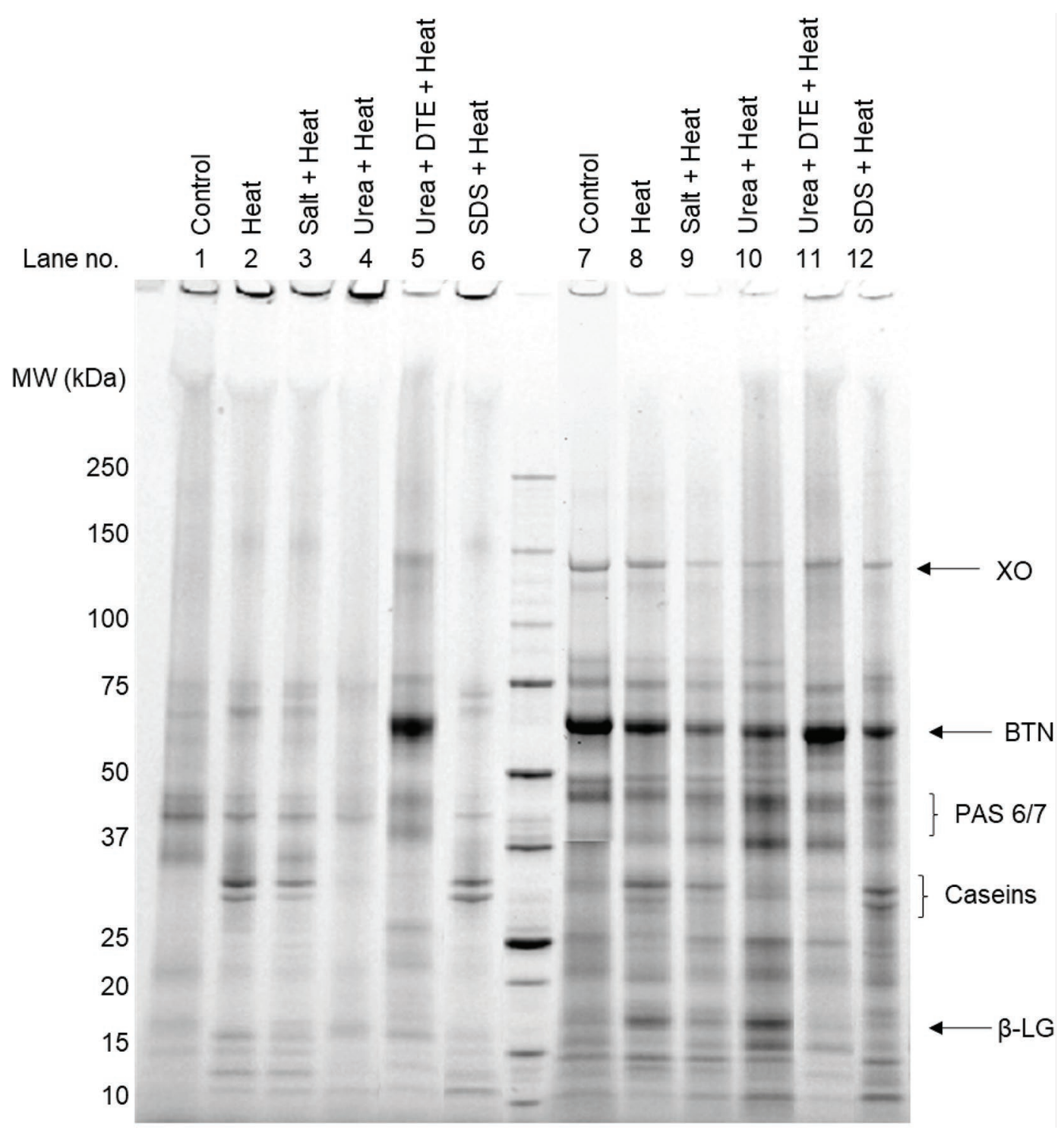

Figure 3. Effect of chaotropic reagents ( $8 M$ urea, $0.2 \%$ SDS, $2 M \mathrm{NaCl}$ ) and the combination of $8 M$ urea and $10 \mathrm{~m} M$ DTE on heat-induced content of $\beta$-LG in milk fat globule membrane material (MFGM). A representative SDS-PAGE is shown with nonreduced (lanes 1-6) and reduced (lanes 7-12) MFGM material. Reduction was done by adding $10 \mathrm{~m} M$ DTE. MW = molecular weight; Std. = molecular weight standard; $\mathrm{XO}=$ xanthine oxidase; BTN = butyrophilin; PAS $6 / 7=$ periodic acid Schiff base 6 and 7 ; DTE $=$ dithioerythritol. 
$(\mathrm{NaCl})$ led to decreased association of $\beta$-LG to MFGM; preventing both hydrogen and disulfide bond formation (urea + DTE) or hydrogen bonds and hydrophobic interactions at once (SDS) completely inhibited the association of $\beta$-LG and MFGM. Overall, our results bring into question the dominant role of thiol-disulfide bond formation in the heat-induced association of $\beta-\mathrm{LG}$ to MFGM. Further studies are needed to consolidate this finding.

\section{ACKNOWLEDGMENTS}

The authors thank Arla Foods for Health Centre (Aarhus N, Denmark), Arla Food Ingredients (Viby J, Denmark), and the Innovation Fund Denmark (Aarhus; Project Infantbrain, Grant file 5158-00014B) for financing this study. The authors have not stated any conflicts of interest.

\section{REFERENCES}

Claumarchirant, L., A. Cilla, E. Matencio, L. M. Sanchez-Siles, P. Castro-Gomez, J. Fontecha, A. Alegría, and M. J. Lagarda. 2016. Addition of milk fat globule membrane as an ingredient of infant formulas for resembling the polar lipids of human milk. Int. Dairy J. 61:228-238. https://doi.org/10.1016/j.idairyj.2016.06.005.

Corredig, M., and D. G. Dalgleish. 1998. Effect of heating of cream on the properties of milk fat globule membrane isolates. J. Agric. Food Chem. 46:2533-2540. https://doi.org/10.1021/jf970944g.

Elfagm, A. A., and J. V. Wheelock. 1978. Interaction of bovine $\alpha$-lactalbumin and $\beta$-lactoglobulin during heating. J. Dairy Sci. 61:28-32. https://doi.org/10.3168/jds.S0022-0302(78)83546-2.

Fox, P. F., T. Uniacke-Lowe, P. L. H. Mcsweeney, and J. A. O'Mahony. 2015. Dairy Chemistry and Biochemistry. 2nd ed. Springer International Publishing, New York, NY.

Gassi, J. Y., M. Blot, E. Beaucher, B. Robert, N. Leconte, B. Camier, F. Rousseau, C. Bourlieu, J. Jardin, V. Briard-Bion, S. Lambert, G. Gésan-Guiziou, C. Lopez, and F. Gaucheron. 2016. Preparation and characterisation of a milk polar lipids enriched ingredient from fresh industrial liquid butter serum: Combination of physicochemical modifications and technological treatments. Int. Dairy J. 52:26-34. https://doi.org/10.1016/j.idairyj.2015.08.012.

Gregersen, S. B., L. Wiking, and M. Hammersh $\varnothing$ j. 2019. Acceleration of acid gel formation by high intensity ultrasound is linked to whey protein denaturation and formation of functional milk fat globule-protein complexes. J. Food Eng. 254:17-24. https://doi .org/10.1016/j.jfoodeng.2019.03.004.

Hansen, S. F., B. Petrat-Melin, J. T. Rasmussen, L. B. Larsen, M. S. Ostenfeld, and L. Wiking. 2018. Placing pasteurisation before or after microfiltration impacts the protein composition of milk fat globule membrane material. Int. Dairy J. 81:35-41. https://doi .org/10.1016/j.idairyj.2017.12.015.

Hoffmann, M.A.M., G. Sala, C. Olieman, and K.G. De Kruif. 1997. Molecular mass distributions of heat-induced $\beta$-lactoglobulin aggregates. J. Agric. Food Chem. 45:2949-2957. https://doi.org/10 $.1021 /$ jf9700788.
Hoffmann, M. A. M., and P. J. J. M. Van Mil. 1997. Heat-induced aggregation of $\beta$-lactoglobulin: Role of the free thiol group and disulfide bonds. J. Agric. Food Chem. 45:2942-2948. https://doi .org/10.1021/jf960789q.

Hong, Y. H., K. Guthy, and H. Klostermeyer. 1984. One the influence of SH-groups in UHT milk during storage. Milchwissenschaft 39:28-30.

Houlihan, A., P. Goddard, S. Nottingham, B. Kitchen, and C. Masters. 1992. Interactions between the bovine milk fat globule membrane and skim milk components on heating whole milk. J. Dairy Res. 59:187-195. https://doi.org/10.1017/S0022029900030430.

Kella, N. K., and J. E. Kinsella. 1988. Structural stability of $\beta$-lactoglobulin in the presence of kosmotropic salts A kinetic and thermodynamic study. Int. J. Pept. Protein Res. 32:396-405. https://doi.org/10.1111/j.1399-3011.1988.tb01274.x.

Kim, H.-H., and R. Jiménez-Flores. 1995. Heat-induced interactions between the proteins of milk fat globule membrane and skim milk. J. Dairy Sci. 78:24-35. https://doi.org/10.3168/jds.S0022 $-0302(95) 76612-7$.

Kitabatake, N., R. Wada, and Y. Fujita. 2001. Reversible conformational change in $\beta$-lactoglobulin A modified with $N$-ethylmaleimide and resistance to molecular aggregation on heating. J. Agric. Food Chem. 49:4011-4018. https://doi.org/10.1021/jf001380a.

Larsen, L. B., A. Wedholm-Pallas, H. Lindmark-Månsson, and A. Andrén. 2010. Different proteomic profiles of sweet whey and rennet casein obtained after preparation from raw versus heat-treated skimmed milk. Dairy Sci. Technol. 90:641-656. https://doi.org/10 $.1051 / \mathrm{dst} / 2010024$

Lee, S. J., and J. W. Sherbon. 2002. Chemical changes in bovine milk fat globule membrane caused by heat treatment and homogenization of whole milk. J. Dairy Res. 69:555-567. https://doi.org/10 .1017/S002202990200571X.

Martin, A. H., M. Nieuwland, and G. A. H. De Jong. 2014. Characterization of heat-set gels from $\mathrm{RuBisCO}$ in comparison to those from other proteins. J. Agric. Food Chem. 62:10783-10791. https://doi .org/10.1021/jf502905g.

Oldfield, D. J., H. Singh, M. W. Taylor, and K. N. Pearce. 2000. Heatinduced interactions of $\beta$-lactoglobulin and $\alpha$-lactalbumin with the casein micelle in pH-adjusted skim milk. Int. Dairy J. 10:509-518. https://doi.org/10.1016/S0958-6946(00)00087-X.

Wada, R., and N. Kitabatake. 2001. B-Lactoglobulin A with N-ethylmaleimide-modified sulfhydryl residue, polymerized through intermolecular disulfide bridge on heating in the presence of dithiothreitol. J Agric. Food Chem. 49:4971-4976. https://doi.org/ 10.1021/jf001457v.

Wada, Y., and B. Lönnerdal. 2015. Effects of industrial heating processes of milk-based enteral formulas on site-specific protein modifications and their relationship to in vitro and in vivo protein digestibility. J. Agric. Food Chem. 63:6787-6798. https://doi.org/ 10.1021/acs.jafc.5b02189.

Wijayanti, H. B., J. Waanders, N. Bansal, and H. C. Deeth. 2015 Identification of the binding of $\beta$-lactoglobulin $(\beta-\mathrm{Lg})$ with sulfhydryl (-SH) blocking reagents by polyacrylamide gel electrophoresis (PAGE) and electrospray ionisation/time of flight-mass spectrometry (ESI/TOF-MS). Lebensm. Wiss. Technol. 63:934-938. https://doi.org/10.1016/J.LWT.2015.04.012.

Ye, A., H. Singh, M. W. Taylor, and S. Anema. 2004. Interactions of whey proteins with milk fat globule membrane proteins during heat treatment of whole milk. Lait 84:269-283. https://doi.org/10 .1051/lait:2004004 